\title{
Lie Symmetry Analysis for Soliton Solutions of Generalised Kadomtsev-Petviashvili-Boussinesq Equation in $(3+1)$-dimensions
}

\author{
VISHAKHA JADAUN ${ }^{1}$, Navnit Jha ${ }^{2}$, and Sachin Ramola ${ }^{3}$ \\ ${ }^{1}$ Netaji Subhas University of Technology \\ ${ }^{2}$ South Asian University \\ ${ }^{3}$ University of Delhi
}

October 29, 2021

\begin{abstract}
The Lie group of infinitesimal transformations technique and similarity reduction is performed for obtaining an exact invariant solution to generalized Kadomstev-Petviashvili-Boussinesq (gKPB) equation in (3+1)-dimensions. We obtain generators of infinitesimal transformations, which provide us a set of Lie algebras. In addition, we get geometric vector fields, a commutator table of Lie algebra, and a group of symmetries. It is observed that the analytic solution (closed-form solutions) to the nonlinear gKPB evolution equations can easily be treated employing the Lie symmetry technique. A detailed geometrical framework related to the nature of the solutions possessing traveling wave, bright and dark soliton, standing wave with multiple breathers, and one-dimensional kink, for the appropriate values of the parameters involved.
\end{abstract}

\section{Hosted file}

V_1.pdf available at https://authorea.com/users/443649/articles/543614-1ie-symmetryanalysis-for-soliton-solutions-of-generalised-kadomtsev-petviashvili-boussinesqequation-in-3-1-dimensions 